\title{
Versatile High-Fidelity Photovoltaic Module Emulation System
}

\author{
Woojoo Lee ${ }^{\dagger}$, Younghyun Kim, Yanzhi Wang ${ }^{\dagger}$, Naehyuck Chang ${ }^{\ddagger}$, Massoud Pedram ${ }^{\dagger}$, \\ and Soohee Han* \\ University of Southern California, CA, USA, :Seoul National University, Korea, \\ *Konkuk University, Korea \\ †\{woojoole, yanzhiwa, pedram\}@usc.edu, $¥\{$ yhkim, naehyuck\}@elpl.snu.ac.kr, \\ *shhan@konkuk.ac.kr
}

\begin{abstract}
Photovoltaic (PV) cells are promising endurable renewable power sources that do not include mechanical components, which are subject to wear and tear. However, actual development of a solarpowered system requires elaborated design processes to find the best setup including location determination and development of a maximum power point tracking method, which requires numerous on-site experiments.

This paper introduces a versatile PV module emulation system, which can cover a range of different PV modules and environmental conditions. We provide an accurate parameter characterization methodology with nonlinear curve fitting to minimize the model discrepancy over the entire operating range. The proposed PV module emulation system includes a pilot PV cell, temperature sensors, an accelerometer, and a magnetic sensor, and provides features for the PV module characterization and emulation modes. Experimental results show significant improvement in the emulation accuracy, which comes from the advanced PV module characterization method as well as high-precision hardware and control.
\end{abstract}

\section{INTRODUCTION}

As a promising and endurable renewable energy source, photovoltaic (PV) cells have been evolving rapidly as semiconductor technology progresses. The quality of the power output is comparable to that of batteries in that it generates a clean DC power output. Despite a major intrinsic drawback in comparison with conventional batteries (i.e., the impedance of the PV module, which is an array of PV cells, is dependent on the solar irradiance level), significant research efforts in the recent past have increased the PV module efficiency and made it one of the most attractive renewable energy sources. Indeed, researchers have developed and successfully applied the maximum power point (MPP) tracking (MPPT) techniques $[1,2,3,4]$.

Solar energy harvesting varies significantly with the time-of-day and location of the PV module. This is because the solar irradiance is continuously changing as a result of these environmental factors, which seriously depreciates the reusability of the data acquired from the PV module previously. Furthermore, PV module

Permission to make digital or hard copies of all or part of this work for personal or classroom use is granted without fee provided that copies are not made or distributed for profit or commercial advantage and that copies bear this notice and the full citation on the first page. To copy otherwise, to republish, to post on servers or to redistribute to lists, requires prior specific permission and/or a fee.

ISLPED'11, August 1-3, 2011, Fukuoka, Japan.

Copyright 2011 ACM X-XXXXX-XX-X/XX/XX ...\$10.00. deployment requires numerous experiments with various kinds of the PV cell and sizes of the PV module, since the produced energy under diverse operating conditions of PV modules are closely related to the unit cell characteristics, array configuration, and its area. As a result, solar energy research has faced a major impediment in that experiments on the target PV module at the specific location involve a significant overhead in terms of human resources and dollar costs.

For the solution to this problem, a rather large number of research results promote "PV module emulation system" $[5,6,7,8$, $9,10,11]$, which reproduce the power-generating behavior of the target PV module under different environmental conditions. The benefits of such emulation systems are not limited to the solar energy, but have been demonstrated for other power sources including batteries [12] and fuel cells [13].

Equivalent circuit models to model a PV module with circuit components such as current source, diode, and resistors have been widely reported $[6,7]$. These references propose equivalent circuit models for the PV modules to mathematically predict their behavior during simulations done in the emulation system. However, mathematical simulation that does not account for real hardware to generate power has a limitation in that we cannot observe the whole system including, for example, DC-DC converters and real load devices. Some recent PV module emulation systems that generate power based on analog circuits have been suggested, including one reported in [5]. Meanwhile, computer-based PV module emulations provide better flexibility based on more elaborated, parameter-adjustable PV models. Such systems are equipped with a controllable power converter such as a bi-directional DC-DC converter [8], a DC-DC buck converter [10], or a full-bridge structure and high frequency transformers [11], which are controlled by the embedded PV model.

Despite the previous works on the PV module simulation and emulation, there is a high potential to further improve the performance and functionality of PV module emulation systems. First of all, the PV module characterization and modeling can be improved by adopting a characterization based on more extensive empirical data. Conventionally, previous emulation systems mostly used a lookup table of V-I curves from measured V-I curves, or used a simplified model derived from a few operating points, such as opencircuit, short-circuit, and MPP. A typical way is to use a numerical method, such as Newton-Raphson method, to extract model parameters for single-diode equivalent circuit $[10,14]$. Such PV module modeling shows a reasonable accuracy for the chosen corner cases. Furthermore, as long as the corner cases can cover the full range of interest, the emulation system guarantees a certain level of accuracy. However, recent research $[3,4,15]$ have decisively shown that not only the MPP but also the whole operating range should be accurately modeled in order to maximize energy efficiency of 


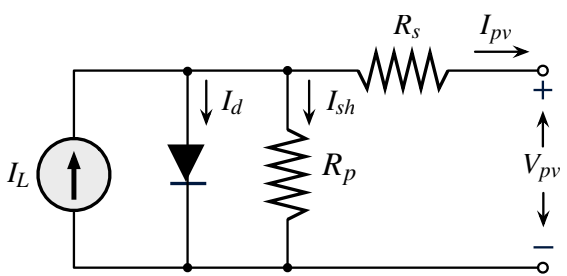

Figure 1: Equivalent circuit model of a PV module.

the PV module. In addition, previous emulation systems rely on the pre-measured or synthetic environmental data. In other words, the irradiance and temperature changes should be given prior to the experiments from previously measured records, or the user has to make the changes manually if necessary. However, there is a strong need to evaluate the developing solar-powered system emulation as if the PV module was actually working under the sunlight at the target location. Consider that we want to find out the optimal location, direction, and tilt of the PV module before deployment, but the conventional emulation systems cannot fulfill such needs because they do not provide such manipulations.

To overcome the previous limitations in PV module emulation systems, our proposed emulation system can measure the current solar irradiance level, temperature, and tilt and direction of the module, by help of a pilot cell, temperature sensors, an accelerometer, and a magnetic sensor. Our emulation system produces power output of the PV module in real time, as well as record the environment profile to reproduce the current PV module behavior in the future. Moreover, we divide the emulation system into two separate units: sensing and emulation units. The sensing unit can be located anywhere (i.e., at the target site, for example, the Mojave desert) without restriction thanks to the wireless communication feature with the emulation unit. We may however place the emulation unit at a different location, for example, in a lab or office in downtown Los Angeles.

We first present a new characterization method to improve the accuracy of the PV circuit model parameters, which is a combination of a numerical method followed by a nonlinear curve fitting. Next, we describe a hardware design of the PV module emulation system to achieve robust performance of real time, model based emulation. Through the experimental results, we prove that it reproduces the whole range of the V-I curve much more accurately than the previous methods. The proposed PV module emulation system can be utilized for designing solar-powered systems whenever and wherever the solar energy is needed with low cost and high accuracy.

\section{PV MODULE CHARACTERIZATION}

\subsection{Photovoltaic Model}

A typical equivalent circuit model of a PV module is shown in Figure 1, with V-I characteristics given by:

$$
\begin{aligned}
I_{p v} & =I_{L}-I_{d}-I_{s h} \\
& =I_{L}(G)-I_{0}(T)\left(e^{\left(V_{p v}+I_{p v} \cdot R_{s}\right)\left(q / A N_{s} k T\right)}-1\right)-\frac{V_{p v}+I_{p v} \cdot R_{s}}{R_{p}},
\end{aligned}
$$

where

$$
I_{L}(G)=\frac{G}{G_{S T C}} \cdot I_{L}\left(G_{S T C}\right)
$$

and

$$
I_{0}(T)=I_{0}\left(T_{S T C}\right)\left(\frac{T}{T_{S T C}}\right)^{3} e^{\left(q E_{g} / A N_{s} k\right)\left(1 / T_{S T C}-1 / T\right)},
$$

where $V_{p v}$ and $I_{p v}$ are the voltage and current of the PV module, respectively. For the parameters, $G$ is the irradiance level; $T$ is the cell temperature; $N_{S}$ is the number of connected cells in series in the PV module; $q$ is the charge of the electron; $E_{g}$ is the energy bandgap and $k$ is Boltzmann's constant. STC stands for standard test condition in which irradiance level is $1000 \mathrm{~W} / \mathrm{m}^{2}$ and temperature is $25^{\circ} \mathrm{C}$. The parameters listed above are either physical constants, environment-related values or configuration parameters. There are still five unknown parameters, commonly not provided by manufacturers, to be determined. These five parameters are the key capable of analytically describing the characteristic of a PV module.

- $I_{L}\left(G_{S T C}\right)$ : the photo-generated current at standard test condition.

- $I_{0}\left(T_{S T C}\right)$ : dark saturation current at standard test condition.

- $R_{S}$ : panel series resistance.

- $R_{p}$ : panel parallel (shunt) resistance.

- $A$ : the diode ideality factor.

A method has been proposed to extract the above-mentioned five parameters from datasheet values in STC, which consist of the open circuit voltage $V_{o c}=\left.V_{p v}\right|_{I_{p v}=0}$, the short circuit current $I_{s c}=I_{p v} \mid V_{p v}=0$, the voltage and current at the MPP $\left(V_{m p p}, I_{m p p}\right)=$ $\arg \max \left(V_{p v} \cdot I_{p v}\right)$, and temperature coefficients [14]. Then a set of $\left(V_{p v}, I_{p v}\right)$

five parameters that determine V-I characteristic are derived from the measured irradiance and temperature by (1), (2) and (3). The output current to the load device is measured, and the microcontroller adjusts the output voltage as derived from the model by (1).

Different from [14], we extract unknown parameters from measured PV module V-I curves at various irradiance levels and temperatures, in which each V-I curve is measured using data acquisition equipment under one specific environmental condition $(G, T)$. Therefore, our parameter extraction method is not confined to only the parameters at STC. Instead, it extracts $I_{L}\left(G_{0}\right)$ and the $I_{0}\left(T_{0}\right)$ flexibly under any environmental condition $\left(G_{0}, T_{0}\right)$ from the measured data. Subsequently, the corresponding parameters at STC, $I_{L}\left(G_{S T C}\right)$ and $I_{0}\left(T_{S T C}\right)$, can be determined using (2) and (3).

Another significant difference from the reference is on the assumption of a priori knowledge of parameter $N_{s}$. The method in [14] assumes that the number of connected PV cells in series in the module, denoted by $N_{s}$, is given a priori, and then parameter $A$ can be derived based on the $N_{s}$ value. However, this assumption is not necessarily valid since $N_{s}$ is usually not specified in datasheets. Therefore, we define a new compound parameter, $N$, as follows:

$$
N=\frac{A N_{s} k T}{q} \text {. }
$$

We extract $N$ from measurement data instead of $A$. In this way, we can emulate the PV module through the extraction of the corresponding $N$ value without prior knowledge on $N_{s}$.

\subsection{PV Module Characterization}

We may extract five unknown parameters $I_{L}\left(G_{0}\right), I_{0}\left(T_{0}\right), R_{S}, R_{p}$ and $N$ from $I_{s c}, V_{o c}, V_{m p p}$ and $I_{m p p}$ for each V-I curve measured under a specific environmental condition $\left(G_{0}, T_{0}\right)$ using a similar method as [14]. This parameter extraction heuristic can be accelerated by Newton-Raphson iteration method. Although such method is quite stable and does not rely heavily on initial values for the 


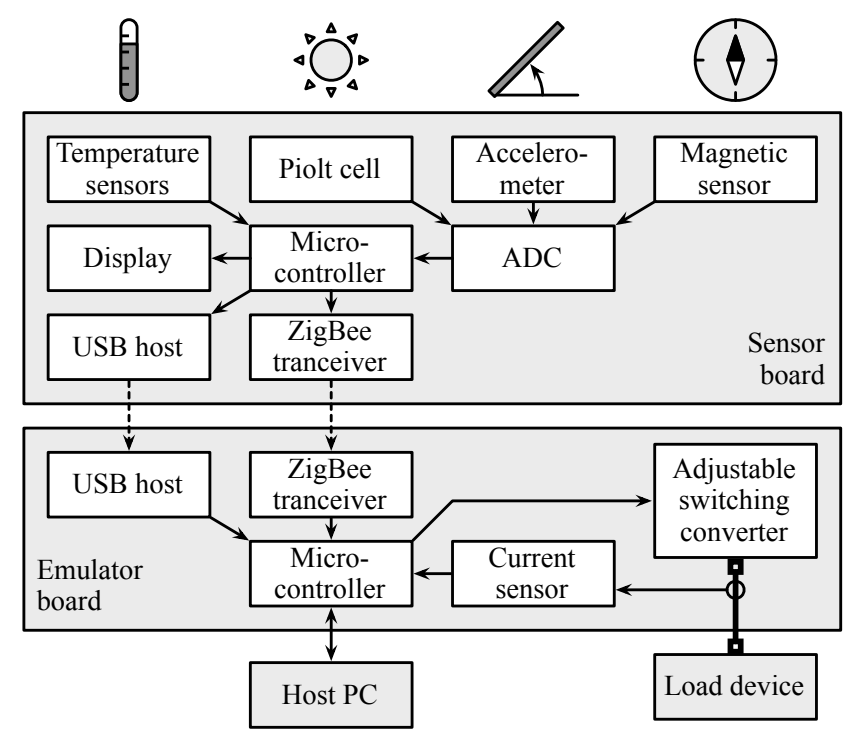

Figure 2: Architecture of the proposed PV module emulation system.

iteration procedure, it does not utilize the whole V-I curve for parameter extraction, and therefore the overall average fitting error cannot be guaranteed to be minimized. Using only a small number of measurement data, not the whole V-I curve, does not really simplify the parameter extraction because we need to measure the whole range anyway to find out the MPP unless we rely on the datasheet provided by the manufacturer. In fact, the fitting errors can be significant in some specific PV module V-I ranges as will be pointed out in Section 4. Hence, such method cannot fulfill the requirement of state-of-the-art researches $[3,4,15]$ that the whole operating range should be accurately modeled for maximizing the energy efficiency.

On the other hand, nonlinear curve fitting algorithm can be adopted here to overcome the shortcoming of the previous method that only some specific points of the whole V-I curve have been used. The fitting parameters depend heavily on the initial values. If the initial values are not properly set, the fitting results obtained may be not optimal nor even feasible. This is because of the fact that nonlinear curve fitting is a highly non-convex optimization problem, and it is likely to be stuck at a local optimal point.

Therefore, we propose to use the parameter extraction heuristic that uses specific points of each V-I curve, accelerated by NewtonRaphson method, in the initial phase. The derived five parameters, i.e., $I_{L}\left(G_{0}\right), I_{0}\left(T_{0}\right), R_{S}, R_{p}$ and $N$, serve as the "proper" initial values in the subsequent least-squares nonlinear curve fitting method based on Levenberg-Marquardt algorithm. Furthermore, we have to also set an upper bound and a lower bound of the fitting parameters, since such bounds also play an important role in the nonlinear curve fitting for acceleration and convergence. One simple, yet effective set of bounds is given by $[\alpha \mathbf{P}, \beta \mathbf{P}]$, where $\mathbf{P}=\left(I_{L}\left(G_{0}\right), I_{0}\left(T_{0}\right), R_{S}, R_{p}, N\right)$ is the derived PV module parameters in the initial phase. With such properly set initial values and upper/lower bounds, nonlinear curve fitting algorithm can find the optimal PV module parameters effectively, taking into account the whole V-I operating range.

We apply the proposed combined parameter extraction method on the measured PV module V-I curves. As pointed out in Section 4 , significant reduction in root mean square (RMS) fitting error can be observed compared with the conventional method which only considers some specific points.

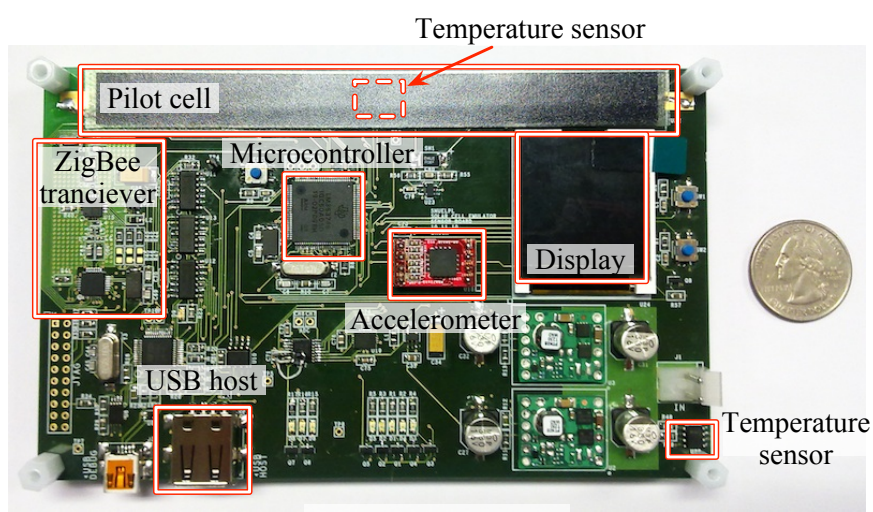

(a) Sensor board

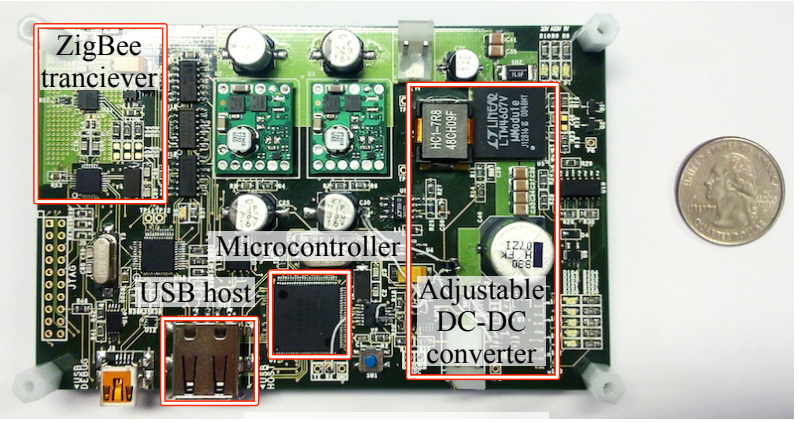

(b) Emulator board

Figure 3: Implemented PV module emulation system boards.

\section{EMULATION SYSTEM}

\subsection{System Architecture}

Figure 2 presents the architecture of the proposed PV module emulation system that is composed of a sensor board and an emulator board. The sensor board consists of a pilot cell, temperature sensors, an accelerometer, and a magnetic sensor for collecting environmental variables including the solar irradiance, ambient temperature, pilot cell temperature, tilt angle of the board, and geographical direction of the board. It shares the acquired data with the emulator board via a ZigBee transceiver or a USB host interface.

The emulator board has a current-controlled switching converter to generate power that corresponds to the received environmental variables based on the the target PV module characterization. We use a PC to communicate with the emulator board in order for monitoring and controlling the entire emulation system through a serial terminal software or a LabView application. This capability provides a user interface to readily configure the emulation system according to the target module, and cope with the environmental variables which even can be selectively overridden by users.

\subsection{Implementation}

Figure 3 shows the implementation of the proposed PV module emulation system boards. We detect the solar irradiance indirectly by measuring $I_{s c}$ of the pilot cell using a current shunt monitor. Measuring $I_{s c}$ instead of $V_{o c}$ provides a more robust measurement because $I_{s c}$ is more sensitive than $V_{o c}$ to change of $G$. We have two temperature sensors of ADT7410 from Analog Devices, one underneath the pilot cell and one at the corner of the sensor board, for the purpose of measuring both ambient and the pilot cell temperatures respectively. The cell temperature influences $V_{o c}$, and so needs to be measured precisely. Using a real PV cell as a pilot cell instead of a photodiode has an advantage that we can measure the 


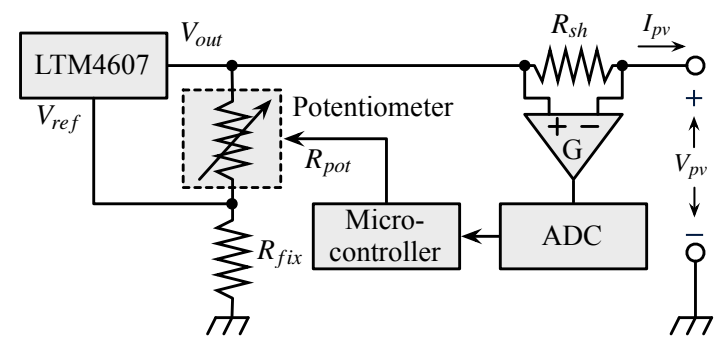

Figure 4: Adjustable switching converter feedback circuit design for the model-based V-I emulation.

cell temperature which may be different from the ambient temperature. The pilot cell temperature sensor is adhered to the pilot cell using thermally conductive epoxy. We can also utilize the ambient temperature data for more detailed analysis, for instance, for considering the wind speed effects [16]. All the measured environmental variables are associated with a time stamp before being sent or saved.

We use an accelerometer and a magnetic sensor to calculate the incidence angle and direction of the sunlight. Honeywell HMC6042 is a two-axis magnetic sensor used as a compass, and Freescale MMA7260Q is a three-axis accelerometer used as a tilt sensor, precisely measuring the tile angle of the sensor board. The angle of incidence is inferred from the output of these sensors and astronomical data. Then the system computes the direction for the optimal direct normal irradiance (DNI). This can be utilized to investigate the solar energy harvesting variation due to the direction, and find the optimal movement of the sun-tracking PV modules [17].

All the analog output voltages from the current shunt monitor, accelerometers, and magnetic sensor are measured using a high precision 12-bit analog to digital converter (ADC) ADS7828 from Texas Instruments. The two temperature sensors and ADC are connected to the CPU using inter-integrated circuit (I2C) serial interface.

The emulator board has an adjustable switching converter which has a wide range of output voltage to mimic the PV module output, based on the PV model introduced in Section 2.1. We use the LTM4607 from Linear Technology as the adjustable switching converter with a digital potentiometer (variable resistor) in the voltage feedback loop as shown in Figure 4. The microcontroller adjusts $R_{p o t}$ according to the following equation:

$$
V_{p v}=V_{\text {out }}-I_{p v} \cdot R_{s h}=V_{r e f} \cdot \frac{R_{f i x}+R_{p o t}}{R_{f i x}}-I_{p v} \cdot R_{s h} .
$$

The digital potentiometer has a 10-bit resolution which can generate 1024 voltage levels with $1 \%$ accuracy. Passive components such as the inductor and output capacitors are carefully selected to have less than a few percent of current ripple. The emulator board may generate up to $5 \mathrm{~A}$ output current or up to $24 \mathrm{~V}$ output voltage which may exceed $100 \mathrm{~W}$, and thus extra care is necessary in the printed circuit board (PCB) design process. Furthermore, the emulator board is composed of various measurement and amplifier circuits which sensitively affect the power emulation quality. We design a four-layer PCB with separated power plains for powersensitive circuitry in such a way that sensors and an RF subsystem are powered by low-noise low drop-out (LDO) voltage regulators. We pay a special attention to the adjustable switching converter in order to reliably provide high power output, including selection of passive components and PCB etch/via design.

The sensor board and emulator board have several means of data transfer. One is IEEE $802.15 \mathrm{ZigBee}$ wireless communication by

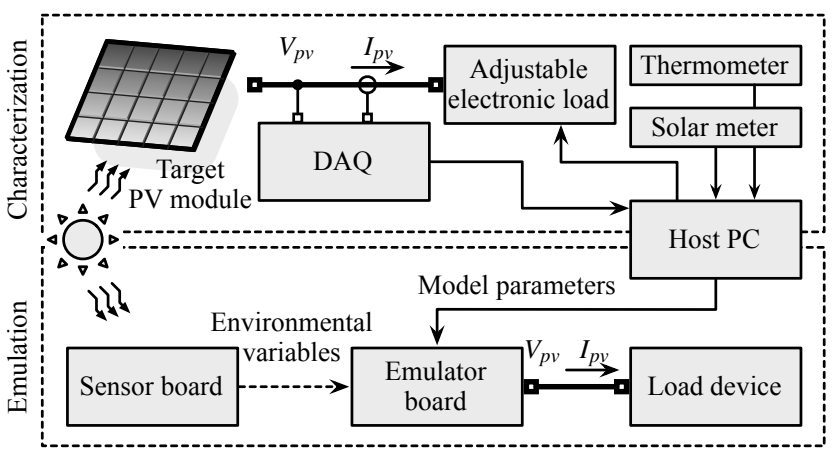

Figure 5: Experimental setup for the characterization and emulation.

which the sensor board transmits time-stamped environment variables in real time to the emulator board. This enables the real-time emulation based on the environment variables measured just now. Alternatively, we can use a USB flash drive to store and restore data. One may want to monitor and record the environmental variables for future use or may want to repeat the experiment under the same condition later. In addition, a serial interface on both boards is used to communicate with a serial terminal software or a LabView application for control and data transfer. We use the Stellaris LM3S3748 microcontroller which includes an ARM CortexM3 core running at $50 \mathrm{MHz}$. This low-power microcontroller has a rich set of peripheral devices including I2C, UART, SPI, and USB serial interfaces, many GPIO pins, and a display controller. We use Micrium MicroC/OS-II RTOS (real-time operating system) to control the system. On top of it, RTOS tasks for measurement, communication, and switching converter control are implemented with consideration on synchronization and priority issues.

\section{EXPERIMENTAL RESULTS}

\subsection{Experimental Setup}

Figure 5 shows the experimental setup for evaluating the proposed characterization method and emulation system. We use an adjustable electronic load and a DAQ (data acquisition) controlled by a PC to measure the V-I characteristics of the target PV module in the characterization step. The electronic load is controlled to sink current ranging from $0 \mathrm{~A}$ (i.e., $V_{p v}=V_{o c}$ ) to $I_{s c}$ (i.e., $V_{p v}=0 \mathrm{~V}$ ). We measure the solar irradiance and temperature also by a standalone solar meter and thermometer, respectively, together with the V-I curve measurement. We use the collected data to extract the PV model parameters as in Section 2.2, which are embedded in the emulator board.

The sensor board provides environmental variables to the emulator board which determines a control set point for the target PV model. The emulator board has a switching power converter circuit which is controlled by the internal feedback loop with the set point, and supplies power output to the load device. The load device may be any real electronics that are intended to be powered by the target PV module. For the verification purpose, we use the adjustable electronic load again to generate a controlled power consumption for emulation system. The target PV module that we use in the experiment is PVL-68, a 68-W flexible PV module from Uni-Solar.

\subsection{Model Evaluation}

First, we show the model accuracy improvement by the proposed characterization method. Table 1 shows the equivalent circuit model parameters introduced in Section 2.1 extracted by the con- 
ventional method and the proposed method, respectively. We apply these parameters to the PV model and derive the V-I curves shown in Figure 6(a). It shows the V-I curves obtained from the measurement compared with the V-I curves derived by the conventional and proposed characterization methods, for three different $G$ values: 840,730 , and $590 \mathrm{~W} / \mathrm{m}^{2}$ at the same temperature $T=27^{\circ} \mathrm{C}$. We see that the proposed method extracts more accurate parameters than the conventional method does. Figures 6(b), (c), and (d) provides a more detailed view of the curves, in a low-voltage range near short-circuit state, a medium-voltage range near the MPP state, and a high-voltage range near open-circuit state, respectively. The V-I curves derived by the proposed method are very close to the measured data, but the others derived by the conventional method have noticeable discrepancy.

Figures 6(c) shows an interesting result regarding the MPP. The MPPs derived by the conventional method are almost the same as the measured data. It is not surprising because what this method does is to fix $V_{o c}, I_{s c}, V_{m p p}$, and $I_{m p p}$ as measured and find the parameters accordingly. This implies that the conventional method highly weights these points than other points. However, as mentioned earlier, not only the accuracy nearby the MPP, but overall accuracy across the whole V-I range should not be excluded when we explore the system in terms of energy efficiency. We do not weight any certain point or range of the V-I curve in the curve fitting, to enhance the overall accuracy. The MPP derived by the proposed method is slightly shifted from the measured data, but accuracy enhancement is observed in the entire range.

It is more clearly seen in Figure 7, which presents the relative and RMS errors of the derived V-I curves compared with the measured data. The curves show the relative error between the measured $I_{p v}$
Table 1: Model parameters extracted by the conventional (NR) method and proposed (CF) method, and derived some $V, I$, and $P$ compared with measured data (Meas.). $T_{0}=27{ }^{\circ} \mathrm{C}$.

\begin{tabular}{|c|c|c|c|c|c|c|c|}
\hline \multirow{2}{*}{$G_{0}$} & \multicolumn{3}{|c|}{ Parameters } & \multicolumn{4}{|c|}{ Derived $V(\mathrm{~V}), I(\mathrm{~A})$, and $P(\mathrm{~W})$} \\
\hline & Value & NR & $\mathrm{CF}$ & Value & Meas. & NR & $\mathrm{CF}$ \\
\hline \multirow{5}{*}{840} & $\overline{I_{L}\left(G_{0}\right)}$ & $4.66 \mathrm{~mA}$ & $4.64 \mathrm{~mA}$ & $V_{o c}$ & 22.50 & 22.51 & 22.49 \\
\hline & $I_{0}\left(T_{0}\right)$ & $11.52 \mathrm{nA}$ & $11.52 \mu \mathrm{A}$ & $I_{s c}$ & 3.85 & 3.85 & 3.87 \\
\hline & $R_{S}$ & $689 \mathrm{~m} \Omega$ & $405 \mathrm{~m} \Omega$ & $V_{m p p}$ & 17.14 & 17.12 & 17.04 \\
\hline & $R_{p}$ & $41.13 \Omega$ & $58.82 \Omega$ & $I_{m p p}$ & 3.22 & 3.23 & 3.24 \\
\hline & $N$ & 37.00 & 57.08 & $P_{m p p}$ & 55.25 & 55.25 & 55.19 \\
\hline \multirow{5}{*}{730} & $I_{L}\left(G_{0}\right)$ & $4.68 \mathrm{~mA}$ & $4.66 \mathrm{~mA}$ & $V_{o c}$ & 22.23 & 22.23 & 22.23 \\
\hline & $I_{0}\left(T_{0}\right)$ & $7.55 \mathrm{nA}$ & $6.98 \mu \mathrm{A}$ & $I_{s c}$ & 3.37 & 3.36 & 3.38 \\
\hline & $R_{s}$ & $761 \mathrm{~m} \Omega$ & $418 \mathrm{~m} \Omega$ & $V_{m p p}$ & 16.99 & 17.02 & 17.02 \\
\hline & $R_{p}$ & $49.63 \Omega$ & $63.91 \Omega$ & $I_{m p p}$ & 2.84 & 2.83 & 2.83 \\
\hline & $N$ & 36.00 & 54.86 & $P_{m p p}$ & 48.24 & 48.25 & 48.14 \\
\hline \multirow{5}{*}{590} & $I_{L}\left(G_{0}\right)$ & $4.87 \mathrm{~mA}$ & $4.86 \mathrm{~mA}$ & $V_{o c}$ & 22.01 & 22.01 & 22.01 \\
\hline & $I_{0}\left(T_{0}\right)$ & $7.61 \mathrm{nA}$ & $2.03 \mu \mathrm{A}$ & $I_{s c}$ & 2.83 & 2.83 & 2.84 \\
\hline & $R_{s}$ & $776 \mathrm{~m} \Omega$ & $472 \mathrm{~m} \Omega$ & $V_{m p p}$ & 17.06 & 17.04 & 17.01 \\
\hline & $R_{p}$ & $53.35 \Omega$ & $62.68 \Omega$ & $I_{m p p}$ & 2.37 & 2.37 & 2.37 \\
\hline & $N$ & 36.00 & 50.28 & $P_{m p p}$ & 40.38 & 40.39 & 40.29 \\
\hline
\end{tabular}

and modeled $I_{p v}$ at $V_{p v} \in\left[0, V_{o c}\right]$ when $G=840 \mathrm{~W} / \mathrm{m}^{2}$ and $T=$ $27{ }^{\circ} \mathrm{C}$. The bars indicate the RMS errors in ten uniform intervals of $V_{p v}$. The error rate of the proposed method is almost negligible when compared with the conventional method across the whole range of $V_{p v}$. It is noticeable that the error rate of the conventional method is small enough only nearby the MPP.

\subsection{PV Module Emulation}

We demonstrate how accurately the proposed PV module em-

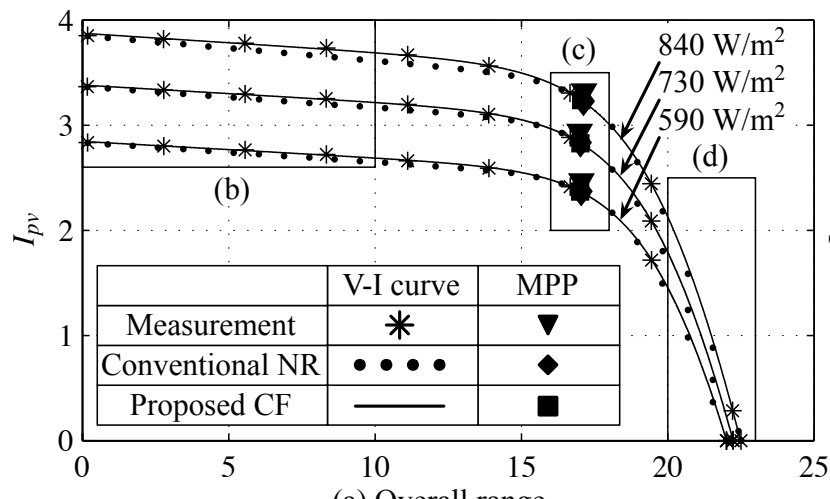

(a) Overall range
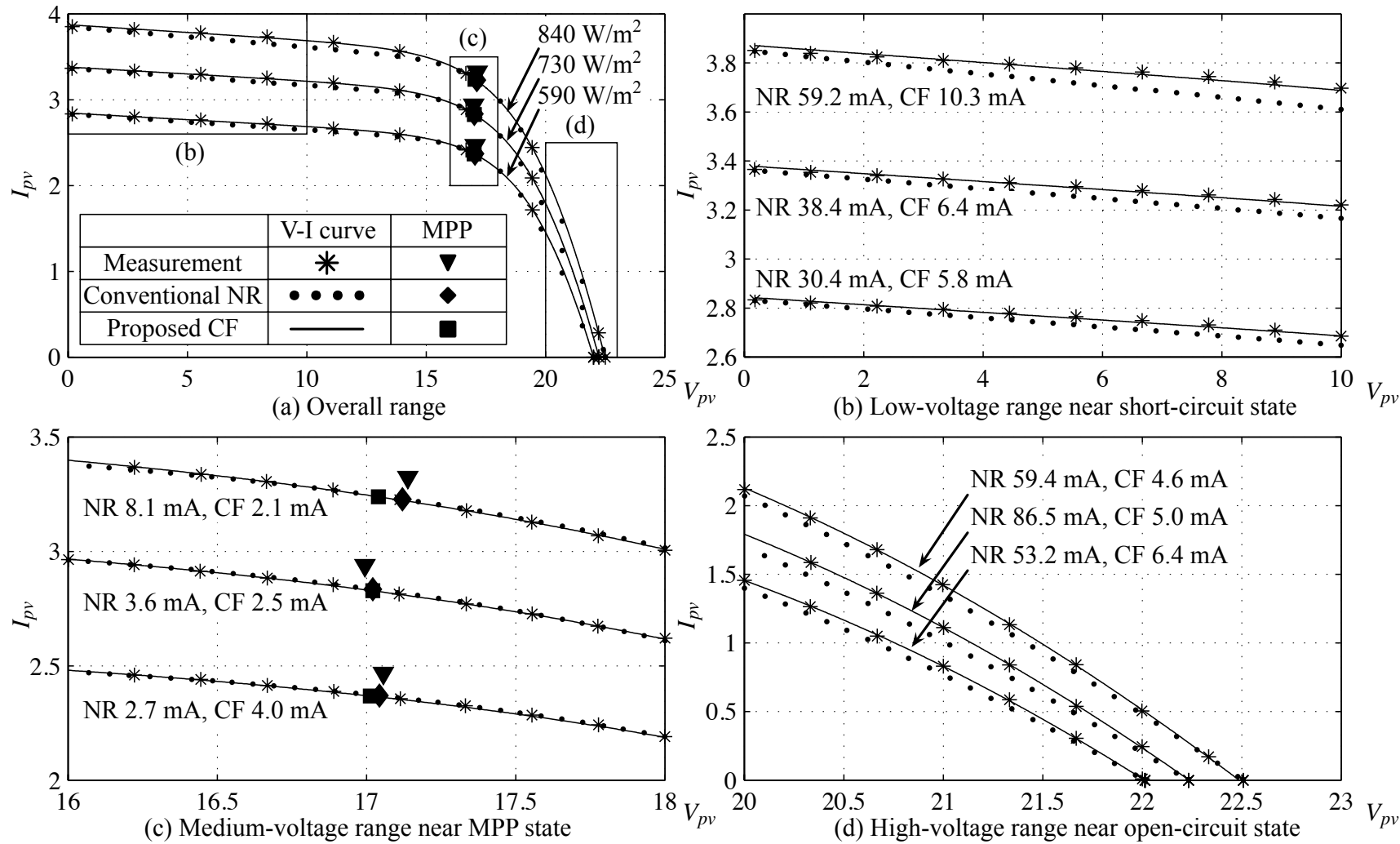

Figure 6: Model comparison with V-I curves in various irradiations. Numbers associated with curves denote the RMS error in each displayed voltage range. $T=27^{\circ} \mathrm{C}$. 


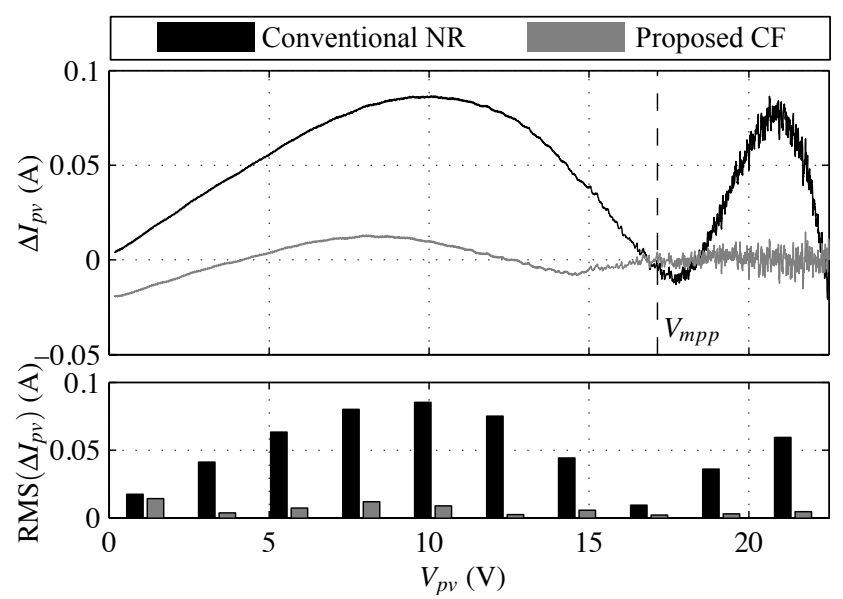

Figure 7: Model errors compared with the measured data. $G=$ $840 \mathrm{~W} / \mathrm{m}^{2}$, and $T=27^{\circ} \mathrm{C}$.

ulation system reproduces the behavior of the modeled PV module. We compare the output $V_{p v}$ and $I_{p v}$ of the emulator board with $V_{p v}$ and $I_{p v}$ measured from the target PV module with varying $G$ over time. Figure 8 shows the variation of $G$ over time and corresponding $V_{p v}$ and $I_{p v}$ measured from the target PV module and the emulator board. A sequence of $G$ collected for three hours, which ranges around $300-850 \mathrm{~W} / \mathrm{m}^{2}$, is supplied to the emulator board. The emulator board is embedded with the PV model based on the parameters extracted in Section 4.2. We apply a constant-power load of $20 \mathrm{~W}$ to the emulator output and measure the voltage and current. The results show that the emulator's output excellently coincides with the target PV module in terms of both $V_{p v}$ and $I_{p v}$ in a wide range of $G$.

\section{CONCLUSIONS}

As efficiency of renewable power systems is significantly affected by environmental variables, extensive on-site experiments are mandatory for successful deployment. Photovoltaic (PV) cells, as representative renewable power sources, are also largely sensitive to the environmental conditions such as solar irradiance and temperature. PV module emulation systems can significantly reduce effort for on-site experiments, but the emulation fidelity should not be compromised for the convenience.

This paper introduces an advanced PV module emulation system with superior accuracy, which comes from nonlinear curve fitting for model parameter extraction, real-time environmental monitoring using rich sensors, wide range of emulation capability with a high-capacity power converter, and better convenience from modular design with wireless communication. Experimental results demonstrate the accuracy of the proposed emulation system in the entire emulation range in terms of the solar irradiance and load current. The proposed emulation system can be utilized to conveniently design solar energy harvesting electronics without being restricted by the time, location and weather conditions for the experiment.

\section{REFERENCES}

[1] T. Esram and P. Chapman, "Comparison of photovoltaic array maximum power point tracking techniques," IEEE T. on Energy Conversion, 2007.

[2] D. Brunelli, C. Moser, L. Thiele, and L. Benini, "Design of a solar-harvesting circuit for batteryless embedded systems," IEEE T. on CAS I, 2009.

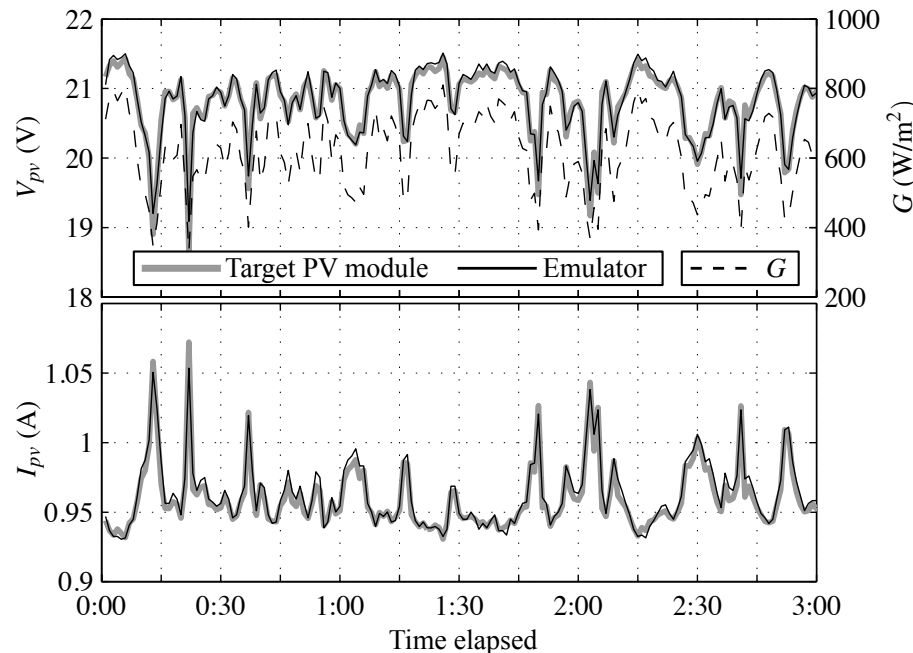

Figure 8: Voltage and current of the PV module emulation system under varying $G$, compared with the results from the target PV module.

[3] Y. Kim, N. Chang, Y. Wang, and M. Pedram, "Maximum power transfer tracking for a photovoltaic-supercapacitor energy system," in ACM/IEEE ISLPED, 2010.

[4] C. Lu, V. Raghunathan, and K. Roy, "Maximum power point considerations in micro-scale solar energy harvesting systems," in IEEE ISCAS, 2010.

[5] H. Nagayoshi, "I-V curve simulation by multi-module simulator using I-V magnifier circuit," Solar Energy Materials and Solar Cells, 2004.

[6] M. Villalva, J. Gazoli, and E. Filho, "Comprehensive approach to modeling and simulation of photovoltaic arrays," IEEE T. on Power Electronics, 2009.

[7] T.-C. Yu and T.-S. Chien, "Analysis and simulation of characteristics and maximum power point tracking for photovoltaic systems," in PEDS, 2009.

[8] P. Sanchis, I. Echeverria, A. Ursua, O. Alonso, E. Gubia, and L. Marroyo, "Electronic converter for the analysis of photovoltaic arrays and inverters," in IEEE PESC, 2003.

[9] J. Enrique, E. Duran, S. de Cardona M, J. Andujar, M. Bohorquez, and J. Carretero, "A new approach to obtain I-V and P-V curves of photovoltaic modules by using DC-DC converters," in IEEE PSC, 2005.

[10] M. Cirrincione, M. Di Piazza, G. Marsala, M. Pucci, and G. Vitale, "Real time simulation of renewable sources by model-based control of DC/DC converters," in IEEE ISIE, 2008.

[11] G. Martin-Segura, J. Lopez-Mestre, M. Teixido-Casas, and A. Sudria-Andreu, "Development of a photovoltaic array emulator system based on a full-bridge structure," in $E P Q U, 2007$.

[12] P. H. Chou, C. Park, J. Park, K. Pham, and J. Liu, "B\#: a battery emulator and power profiling instrument," in ACM/IEEE ISLPED 2003.

[13] G. Marsala, M. Pucci, G. Vitale, M. Cirrincione, and A. Miraoui, "A prototype of a fuel cell pem emulator based on a buck converter," Applied Energy, 2009.

[14] D. Sera, R. Teodorescu, and P. Rodriguez, "PV panel model based on datasheet values," in IEEE ISIE, 2007.

[15] M. Alonso-García, J. Ruiz, and F. Chenlo, "Experimental study of mismatch and shading effects in the I-V characteristic of a photovoltaic module," Solar Energy Materials and Solar Cells, 2006.

[16] E. Skoplaki, A. Boudouvis, and J. Palyvos, "A simple correlation for the operating temperature of photovoltaic modules of arbitrary mounting," Solar Energy Materials and Solar Cells, 2008.

[17] R.-J. Wai, W.-H. Wang, and C.-Y. Lin, "High-performance stand-alone photovoltaic generation system," IEEE T. on Industrial Electronics, 2008 